\title{
A Calculus for Power-Aware Multicast Communications in Ad Hoc Networks
}

\author{
Lucia Gallina and Sabina Rossi \\ Dipartimento di Informatica, Università Ca' Foscari, Venezia (Italy) \\ \{lgallina, srossi\}@dsi.unive.it
}

\begin{abstract}
We present $\mathrm{CMN}^{\#}$, a process calculus for formally modelling and reasoning about Mobile Ad Hoc Networks (MANETs) and their protocols. Our calculus naturally captures essential characteristics of MANETs, including the ability of a MANET node to broadcast a message to any other node within its physical transmission range, and to move in and out of the transmission range of other nodes in the network. In order to reason about cost-effective ad hoc routing protocols, we also allow unicast and multicast communications as well as the possibility for a node to control the transmission radius of its communications. We show how to use our calculus to prove some useful connectivity properties which can be exploited to achieve low-cost routing solutions.
\end{abstract}

\section{Introduction}

A Mobile Ad Hoc Network (MANET) is a self-configuring network of mobile devices connected by wireless links. Each device in a MANET is free to move independently in any direction, and will therefore change its links to other devices frequently. Each node must forward traffic unrelated to its own usage, and then be a router. The primary challenge in building a MANET is equipping each device to continuously maintain the information required to properly route traffic. The devices communicate with each other via radio transceivers through the protocol IEEE 802.11 (WiFi) 9]. This type of communication has a physical scope, because a radio transmission spans over a limited area. Different protocols are evaluated based on the packet drop rate, the routing overhead, the power control, and other measures.

As mobile ad hoc networks communicate in a self organized way without depending on any fixed infrastructure, they are the best solution for various applications, ranging from the monitoring of herds of animals to supporting communications in military battlefields and civilian disaster recovery scenarios. Many of these applications require that nodes be mobile and be deployed with little network planning. The mobility of nodes limits their size, which in turn limits the energy reserves available to them. Moreover, in wireless networks, bandwidth is precious and scarce. Thus energy and bandwidth conservation is a key requirement in the design of MANETs.

Routing in ad hoc networks faces extreme challenges due to node mobility/dynamics and limited communication resources (energy and bandwidth). 
Table 1. Syntax

\begin{tabular}{clll}
\hline Networks & & Processes & \\
$\mathrm{M}, \mathrm{N}::=\mathbf{0}$ & Empty network & $\mathrm{P}, \mathrm{Q}, \mathrm{R}::=\mathbf{0}$ & Inactive process \\
$\left|M_{1}\right| M_{2}$ & Parallel composition & $\mid c(\tilde{x}) . P$ & Input \\
$\mid(\nu c) M$ & Channel restriction & $\mid \bar{c}_{L}\langle\tilde{w}\rangle . P$ & Output \\
$\mid n[P]_{l, r}^{\mu}$ & Node (or device) & $\mid\left[w_{1}=w_{2}\right] P, Q$ & Matching \\
& & $\mid A\langle\tilde{w}\rangle$ & Recursion \\
\hline
\end{tabular}

The routing protocols for mobile ad hoc networks have to adapt quickly to frequent and unpredictable topology changes and must be parsimonious of communication and processing resources. Moreover, since radio signals are likely to overlap with others in a geographical area, a straightforward broadcasting by flooding is usually very costly and will result in serious redundancy, contention, and collision. For this reason, modern ad hoc routing protocols use unicast and multicast communications to reduce the number of control packets (see, e.g., $\left.\begin{array}{llll}\hline & 1 & 2 & 7\end{array}\right]$ ). In addition, power aware protocols reduce the total energy consumption by adjusting each node's transmission power (e.g., radius) just enough to reach up to the intended receivers only (see, e.g., [10]).

In this paper we present a calculus, named $\mathrm{CMN}^{\#}$, for formally modelling and reasoning about mobile ad hoc networks and their protocols. This is an extension of CMN (Calculus of Mobile Ad Hoc Networks), proposed by Merro in 44. It naturally captures essential characteristics of MANETs, including the ability of a MANET node to broadcast a message to any other node within its physical transmission range, and to move in and out of the transmission range of other nodes in the network. In our model the connectivity of a node is represented by a location and a transmission radius. Broadcast communications are limited to the transmission cell of the sender. Unicast and multicast communications are modelled by specifying, for each output action, the addresses of the intended recipients of the message. Moreover, the arbitrary and unexpected connections and disconnections of nodes as well as the possibility for a node to dynamically adjust its transmission power are represented by enabling nodes to modify the corresponding transmission radius through internal actions.

We show how to use our calculus to prove some useful connectivity properties of MANETs which can be exploited to control power/energy consumption. For instance, we can determine the minimum transmission radius ensuring the connectivity of a node with all the intended recipients of its transmissions, thus reducing power consumption.

\section{The Calculus}

We introduce the language CMN\#, an extension of CMN (Calculus of Mobile Ad Hoc Networks) 4], that models mobile ad hoc networks as a collection of nodes, running in parallel, and using channels to broadcast messages. Our calculus extends CMN to support multicast and unicast communications. Moreover, 
it allows one to model the arbitrary and unexpected connections and disconnections of nodes in a network as well as the possibility for a node to administrate power control by choosing the optimal transmission radius to communicate with the desired receivers.

We use letters $c$ and $d$ for channels; $m$ and $n$ for nodes; $l, k$ and $h$ for locations; $r$ for transmission radii; $x, y$ and $z$ for variables. Closed values contain nodes, locations, transmission radii and any basic value (booleans, integers, ...). Values include also variables. We use $u$ and $v$ for closed values and $w$ for (open) values. We denote by $\tilde{v}, \tilde{w}$ tuples of values.

The syntax of CMN\# is shown in Table 11. This is defined in a two-level structure: the lower one for processes, the upper one for networks. Networks are collections of nodes (which represent devices), running in parallel, using channels to communicate messages. As usual, $\mathbf{0}$ denotes the empty network and $M_{1} \mid M_{2}$ represents the parallel composition of two networks. The restriction in $(\nu c) M$ acts as the standard CCS restriction (i.e., it does not perform any channel creation). Processes are sequential and live within the nodes. Process $\mathbf{0}$ denotes the inactive process. Process $c(\tilde{x}) . P$ can receive a tuple $\tilde{w}$ of (closed) values via channel $c$ and continue as $P\{\tilde{w} / \tilde{x}\}$, i.e., as $P$ with $\tilde{w}$ substituted for $\tilde{x}$ (where $|\tilde{x}|=|\tilde{w}|)$. Process $\bar{c}_{L}\langle\tilde{w}\rangle \cdot P$ can send a tuple of (closed) values $\tilde{w}$ via channel $c$ and continue as $P$. The $\operatorname{tag} L$ is used to maintain the set of locations of the intended recipients: $L=\infty$ represents a broadcast transmission, while a finite set of locations $L$ denotes a multicast communication (unicast if $L$ is a singleton). Syntactically, $L$ may be a variable, but it must be a set of locations when the output prefix is ready to fire. Process $\left[w_{1}=w_{2}\right] P, Q$ behaves as $P$ if $w_{1}=w_{2}$, and as $Q$ otherwise. We write $A\langle\tilde{w}\rangle$ to denote a process defined via a (possibly recursive) definition $A(\tilde{x}) \stackrel{\text { def }}{=} P$, with $|\tilde{x}|=|\tilde{w}|$, where $\tilde{x}$ contains all channels and variables that appear free in $P$.

Each node, if connected, has a location and a transmission radius. Nodes cannot be created or destroyed. We write $n[P]_{l, r}^{\mu}$ for a node named $n$ (this is the logic location of the device in the network), located at $l$, with transmission radius $r$, mobility tag $\mu$, and executing a process $P$. The tag $\mu$ is $\mathrm{m}$ for mobile nodes, and $\mathrm{s}$ for stationary nodes; $l$ denotes the physical location of the node. To each node $n$ is associated a maximum transmission radius $r_{n}$; nodes may control power consumption by dynamically adjusting their transmission radius $r$ provided that $r \in\left[0, r_{n}\right]$. Notice that if $r=0$ then the node is disconnected.

In the process $c(\tilde{x}) . P$, the tuple $\tilde{x}$ is bound in $P$; while in $(\nu c) M$, the channel name $c$ is bound in $M$. We denote by $f v(\cdot)$ and $f c(\cdot)$ free variables and channels, respectively, and identify processes and networks up to $\alpha$-conversion. Parallel composition of networks has lower precedence with respect to restriction. We denote by $\prod_{i \in I} M_{i}$ the parallel composition of networks $M_{i}$, for $i \in I$. We write $(\nu \tilde{c}) M$ as an abbreviation for $\left(\nu c_{1}\right) \ldots\left(\nu c_{k}\right) M$. To denote unicast communication, we write $c_{l}$ for $c_{\{l\}}$. We write $\bar{c}_{L}\langle w\rangle$ for $\bar{c}_{L}\langle w\rangle . \mathbf{0}, \mathbf{0}$ for $n[\mathbf{0}]_{l, r}^{\mu}$ and $\left[w_{1}=w_{2}\right] P$ for $\left[w_{1}=w_{2}\right] P, \mathbf{0}$. We assume that there are no free variables in a network (while there can be free channels). Moreover, we assume that networks are wellformed, i.e., each node identifier is unique and the corresponding transmission 
Table 2. Structural Congruence

\begin{tabular}{ll}
\hline$n[[v=v] P, Q]_{l, r}^{\mu} \equiv n[P]_{l, r}^{\mu} \quad$ & (Struct Then) \\
$n\left[\left[v_{1}=v_{2}\right] P, Q\right]_{l, r}^{\mu} \equiv n[Q]_{l, r}^{\mu} \quad v_{1} \neq v_{2}$ & (Struct Else) \\
$n[A\langle\tilde{v}\rangle]_{l, r}^{\mu} \equiv n[\{\tilde{v} / \tilde{x}\} P]_{l, r}^{\mu} \quad$ if $A(\tilde{x}) \stackrel{\text { def }}{=} P \wedge|\tilde{x}|=|\tilde{v}|$ & (Struct Rec) \\
$M|N \equiv N| M$ & (Struct Par Comm) \\
$(M \mid N)\left|M^{\prime} \equiv M\right|\left(N \mid M^{\prime}\right)$ & (Struct Par Assoc) \\
$M \mid \mathbf{0} \equiv M$ & (Struct Zero Par) \\
$(\nu c) \mathbf{0} \equiv \mathbf{0}$ & (Struct Zero Res) \\
$(\nu c)(\nu d) M \equiv(\nu d)(\nu c) M$ & (Struct Res Res) \\
$(\nu c)(M \mid N) \equiv M \mid(\nu c) N \quad$ If $c \notin f c(M)$ & (Struct Res Par) \\
$M \equiv M \quad$ if $M \equiv N$ & (Struct Refl) \\
$N \equiv M \quad$ (Struct Symm) \\
$M \equiv M^{\prime \prime}$ if $M \equiv M^{\prime} \wedge M^{\prime} \equiv M^{\prime \prime}$ & (Struct Trans) \\
$M\left|M^{\prime} \equiv N\right| M^{\prime} \forall M^{\prime}$ if $M \equiv N$ & (Struct Cxt Par) \\
$(\nu c) M \equiv(\nu c) N \quad \forall c$ if $M \equiv N$ & (Struct Cxt Res) \\
\hline
\end{tabular}

radius is compatible with the node's power capacity. Formally, a network $M \equiv$ $n_{1}\left[P_{1}\right]_{l_{1}, r_{1}}^{\mu_{1}}\left|n_{2}\left[P_{2}\right]_{l_{2}, r_{2}}^{\mu_{2}} \ldots\right| n_{k}\left[P_{k}\right]_{l_{k}, r_{k}}^{\mu_{k}}$ is well-formed if for $i, j \in\{1, \ldots, k\}$ it holds that $n_{i} \neq n_{j}$ when $i \neq j$ and $r_{i} \in\left[0, r_{n_{i}}\right]$.

Reduction Semantics. The dynamics of the calculus is specified by the reduction relation over networks $(\rightarrow)$, described in Table 3 . As usual, it relies on an auxiliary relation, called structural congruence $(\equiv)$, defined in Table 2 . We assume the possibility of comparing locations in order to determine whether a node lies or not within the transmission cell of another node. This is done through function $d(\cdot, \cdot)$ which takes two locations and returns their distance.

Rule (R-Bcast) models the transmission of a tuple $\tilde{v}$ through a channel $c_{L}$. The set $L$ associated to channel $c$ indicates the locations of the intended recipients, even if broadcast communications are indeed performed. If $L=\infty$ then the recipients set is the whole network (broadcast transmission), while a finite set $L$ (resp., a singleton) is used to denote a multicast (resp., a unicast) communication. In our calculus transmission is a non-blocking action: transmission proceeds even if there are no nodes listening for messages. The messages transmitted will be received only by those nodes which lie in the transmission area of the sender. It may occur that some receivers within the range of the transmitter do not receive the message. This may be due to several reasons that concern the instability and dynamism of the network. In terms of observation this corresponds to a local activity of the network which an observer is not party to. Rule (R-Rad) models the possibility for a node $n$ to control power consumption by changing its transmission radius $r$ into $r^{\prime}$ provided that $r^{\prime} \in\left[0, r_{n}\right]$. Rule (R-Move) models arbitrary and unpredictable movements of mobile nodes. $\delta$ denotes the maximum distance that a node can cover in a computational step. Notice that a node is disconnected when its radius is set to 0 . We denote by $\longrightarrow^{*}$ the reflexive and transitive closure of $\longrightarrow$. 
Table 3. Reduction Semantics

$$
\begin{aligned}
& \text { (R-Bcast) } \frac{r \neq 0, \forall i \in I . d\left(l, l_{i}\right) \leq r, r_{i} \neq 0,\left|\tilde{x}_{i}\right|=|\tilde{v}|}{n\left[\bar{c}_{L}\langle\tilde{v}\rangle \cdot P\right]_{l, r}^{\mu}\left|\prod_{i \in I} n_{i}\left[c\left(\tilde{x}_{i}\right) \cdot P_{i}\right]_{l_{i}, r_{i}}^{\mu_{i}} \rightarrow n[P]_{l, r}^{\mu}\right| \prod_{i \in I} n_{i}\left[P_{i}\left\{\tilde{v} / \tilde{x}_{i}\right\}\right]_{l_{i}, r_{i}}^{\mu_{i}}} \\
& (\mathrm{R}-\mathrm{Rad}) \frac{r^{\prime} \in\left[0, r_{n}\right]}{n[P]_{l, r}^{\mu} \rightarrow n[P]_{l, r^{\prime}}^{\mu}} \\
& \text { (R-Move) } \frac{d(l, k) \leq \delta}{n[P]_{l, r}^{\mathrm{m}} \rightarrow n[P]_{k, r}^{\mathrm{m}}} \\
& (\mathrm{R}-\mathrm{Par}) \frac{M \rightarrow M^{\prime}}{M\left|N \rightarrow M^{\prime}\right| N} \\
& (\mathrm{R}-\mathrm{Res}) \frac{M \rightarrow M^{\prime}}{(\nu c) M \rightarrow(\nu c) M^{\prime}} \\
& \text { (R-Struct) } \frac{M \equiv N N \rightarrow N^{\prime} N^{\prime} \equiv M^{\prime}}{M \rightarrow M^{\prime}}
\end{aligned}
$$

Behavioral Semantics. The central actions of our calculus are transmission and reception of messages. However, only the transmission of messages (over unrestricted channels) can be observed. An observer cannot be sure whether a recipient actually receives a given value. Instead, if a node receives a message, then surely someone must have sent it. As usual, we adopt the term barb as a synonymous of observable. In our definition of barb a transmission is considered an observable action only if at least one location in the set of the intended recipients is able to receive the message.

Definition 1 (Barb). We write $M \downarrow_{c}$ if $M \equiv(\nu \tilde{d})\left(n\left[\bar{c}_{L}\langle\tilde{v}\rangle \cdot P\right]_{l, r}^{\mu} \mid M^{\prime}\right)$, with $c \notin \tilde{d}$ and $\exists k \in L \wedge d(l, k) \leqslant r$. We write $M \Downarrow_{c}$ if $M \longrightarrow^{*} M^{\prime} \downarrow_{c}$.

Notice that, if $M \equiv(\nu \tilde{d})\left(n\left[\bar{c}_{L}\langle\tilde{v}\rangle \cdot P\right]_{l, r}^{\mu} \mid M^{\prime}\right)$ and $M \downarrow_{c}$ then at least one of the locations in $L$ is actually able to receive the message.

To define our observation equivalence we will ask for the largest relation which satisfies the following properties. Let $\mathcal{R}$ be a relation over networks:

Barb preservation. $\mathcal{R}$ is barb preserving if $M \mathcal{R} N$ and $M \downarrow_{c}$ implies $N \Downarrow_{c}$.

Reduction closure. $\mathcal{R}$ is reduction closed if $M \mathcal{R} N$ and $M \longrightarrow M^{\prime}$ implies that there exists $N^{\prime}$ such that $N \longrightarrow{ }^{*} N^{\prime}$ and $M^{\prime} \mathcal{R} N^{\prime}$.

Contextuality. $\mathcal{R}$ is contextual if $M \mathcal{R} N$ implies $\mathcal{C}[M] \mathcal{R} \mathcal{C}[N]$ for any context $\mathcal{C}[\cdot]$, where a context is a network term with a hole $[\cdot]$ defined by:

$$
\mathcal{C}[\cdot]::=[\cdot]|[\cdot]| M|M|[\cdot] \mid(\nu c)[\cdot]
$$

Definition 2 (Reduction barbed congruence). Reduction barbed congruence, written $\cong$, is the largest symmetric relation over networks, which is reduction closed, barb preserving, and contextual. 
Table 4. LTS rules for Processes

(Output) $\frac{-}{\bar{c}_{L}\langle\tilde{v}\rangle \cdot P \stackrel{\bar{c}_{L} \tilde{v}}{\rightarrow} P} \quad$ (Input) $\frac{-}{c(\tilde{x}) \cdot P \stackrel{c \tilde{v}}{\rightarrow} P\{\tilde{v} / \tilde{x}\}}$
(Then) $\frac{P \stackrel{\eta}{\rightarrow} P^{\prime}}{[\tilde{v}=\tilde{v}] P, Q \stackrel{\eta}{\rightarrow} P^{\prime}} \quad($ Else $) \frac{Q \stackrel{\eta}{\rightarrow} Q^{\prime} \quad \tilde{v_{1}} \neq \tilde{v}_{2}}{\left[\tilde{v_{1}}=\tilde{v_{2}}\right] P, Q \stackrel{\eta}{\rightarrow} Q^{\prime}}$
$(\operatorname{Rec}) \frac{P\{\tilde{v} / \tilde{x}\} \stackrel{\eta}{\rightarrow} P^{\prime} A(\tilde{x}) \stackrel{\text { def }}{=} P}{A\langle\tilde{v}\rangle \stackrel{\eta}{\rightarrow} P^{\prime}}$

\section{Bisimulation-Based Proof Method}

In this section we develop a proof technique for the relation $\cong$. More precisely, we define a LTS semantics for CMN\# terms, which is built upon two sets of rules: one for processes and one for networks. Table 4 presents the LTS rules for processes. Transitions are of the form $P \stackrel{\eta}{\rightarrow} P^{\prime}$, where $\eta$ ranges over input and output actions of the form $c \tilde{v}$ and $\bar{c}_{L} \tilde{v}$, respectively. Table 5 presents the LTS rules for networks. Transitions are of the form $M \stackrel{\gamma}{\rightarrow} M^{\prime}$, where $\gamma$ is as follows:

$$
\gamma::=c ? \tilde{v} @ l\left|c_{L} ! \tilde{v}[l, r]\right| c ! \tilde{v} @ K \mid \tau .
$$

Rules for processes are simple and they do not need deeper explanations. Let us illustrate the rules for networks. Rule (Snd) models the sending, with transmission radius $r$, of the tuple $\tilde{v}$ through channel $c$ to a specific set $L$ of recipients, while rule (Rcv) models the reception of $\tilde{v}$ at $l$ via channel $c$. Rule (Bcast) models the broadcast message propagation: all the nodes lying within the transmission cell of the sender may receive the message, regardless of the fact that they are in $L$. Rule (Obs) models the observability of a transmission: every output action may be detected (and hence observed) by any node located within the transmission cell of the sender. The action $c ! \tilde{v} @ K$ represents the transmission of the tuple $\tilde{v}$ of messages via $c$ to a set $K$ of recipients in $L$, located within the transmission cell of the transmitter. When $K \neq \emptyset$ this is an observable action corresponding to the barb $\downarrow_{c}$. Rule (Lose) models both message loss and a local activity of the network which an observer is not party to. As usual, $\tau$-transitions are used to denote non-observable actions. Rule (Move) models migration of a mobile node from a location $l$ to a new location $k$, where $\delta$ represents the maximum distance that a node can cover in a single computational step. Rule (Rad) models the possibility for a node $n$ to change its transmission radius, provided that it is within $\left[0, r_{n}\right]$. Finally (Par) and (Res) are standard. Notice that since we do not transmit channels, in our calculus there is no scope extrusion.

The following relationships between the LTS semantics and the reduction one hold. 
Table 5. LTS rules for Networks

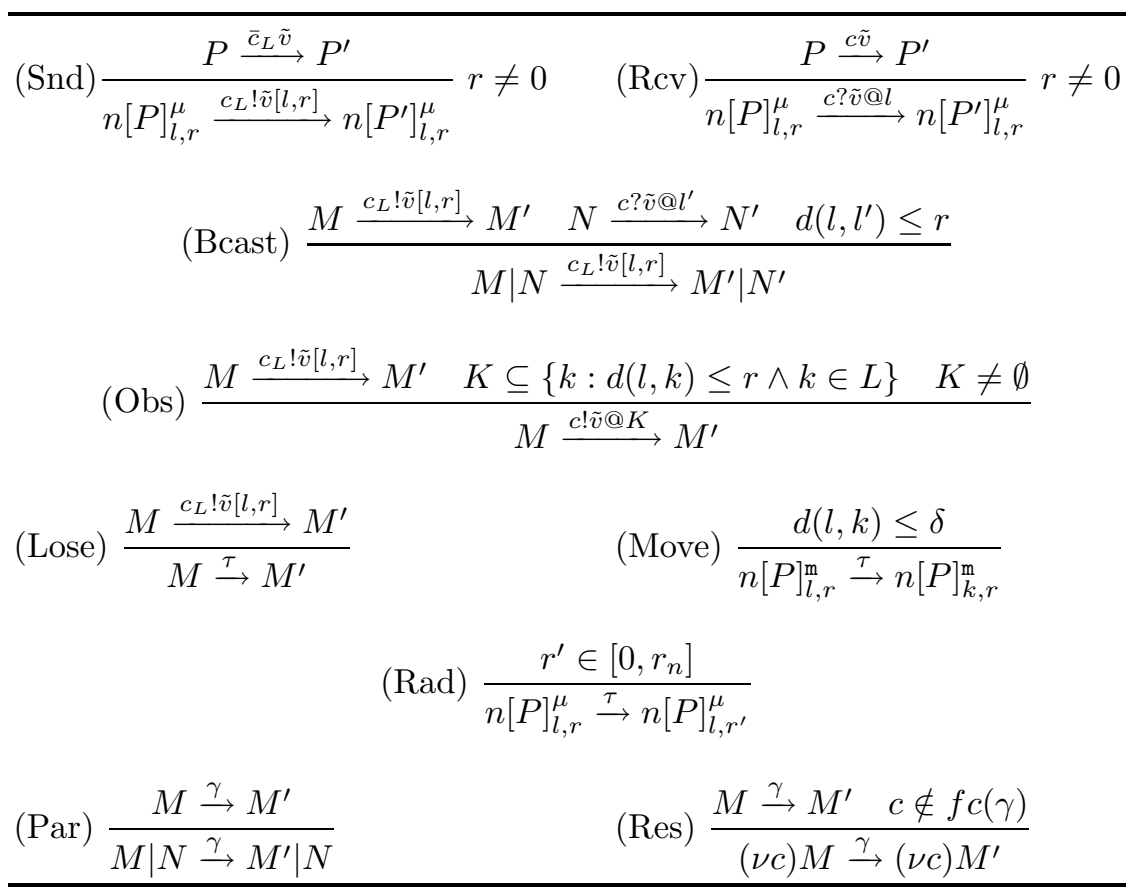

Lemma 1. Let $M$ be a network.

1. If $M \stackrel{c ? \tilde{v} @ l}{\longrightarrow} M^{\prime}$, then there are $n, P, \mu, l, r, M_{1}$ and $\tilde{d}$, with $c \notin \tilde{d}$, such that $M \equiv(\nu \tilde{d})\left(n[c(\tilde{x}) \cdot P]_{l, r}^{\mu} \mid M_{1}\right)$ and $M^{\prime} \equiv(\nu \tilde{d})\left(n[P\{\tilde{v} / \tilde{x}\}]_{l, r}^{\mu} \mid M_{1}\right)$.

2. If $M \stackrel{c_{L} ! \tilde{v}[l, r]}{\longrightarrow} M^{\prime}$, then there are $n, P, \mu, l, r, M_{1}, I$ (possibly empty), and $\tilde{d}$, with $c \notin \tilde{d}$, and $n_{i}, P_{i}, \mu_{i}, l_{i}, r_{i}$, with $d\left(l, l_{i}\right) \leq r$ for all $i \in$ $I$, such that: $M \equiv(\nu \tilde{d})\left(n\left[\bar{c}_{L}\langle\tilde{v}\rangle \cdot P\right]_{l, r}^{\mu}\left|\prod_{i \in I} n_{i}\left[c\left(\tilde{x}_{i}\right) . P_{i}\right]_{l_{i}, r_{i}}^{\mu_{i}}\right| M_{1}\right)$ and $M^{\prime} \equiv$ $(\nu \tilde{d})\left(n[P]_{l, r}^{\mu}\left|\prod_{i \in I} n_{i}\left[P_{i}\left\{\tilde{v} / \tilde{x}_{i}\right\}\right]_{l_{i}, r_{i}}^{\mu_{i}}\right| M_{1}\right)$.

Lemma 2. Let $M$ be a network. It holds that $(i) M \downarrow_{c}$ if and only if $M \stackrel{c ! \tilde{v} @ K}{\longrightarrow}$ for some tuple of values $\tilde{v}$ and set of locations $K$; (ii) if $M \stackrel{\tau}{\rightarrow} M^{\prime}$ then $M \longrightarrow M^{\prime}$; (iii) if $M \longrightarrow M^{\prime}$ then $M \stackrel{\tau}{\longrightarrow} \equiv M^{\prime}$.

We now introduce a labelled bisimilarity that is a complete characterization of our notion of reduction barbed congruence. We adopt the metavariable $\alpha$ to range over those actions that will be used in the definition of labelled bisimilarity:

$$
\alpha::=c ? \tilde{v} @ l|c ! \tilde{v} @ K| \tau .
$$

Since we are interested in weak behavioral equivalences, that abstract over $\tau$ actions, we introduce the notion of weak action. We denote by $\Rightarrow$ the reflexive and transitive closure of $\stackrel{\tau}{\longrightarrow}$; we use $\stackrel{c ? \tilde{v} @ l}{\Longrightarrow}$ to denote $\Rightarrow \stackrel{c ? \tilde{v} @ l}{\longrightarrow} \Rightarrow$; we use $\stackrel{c ? \tilde{v} @ F}{\Longrightarrow}$ to denote 


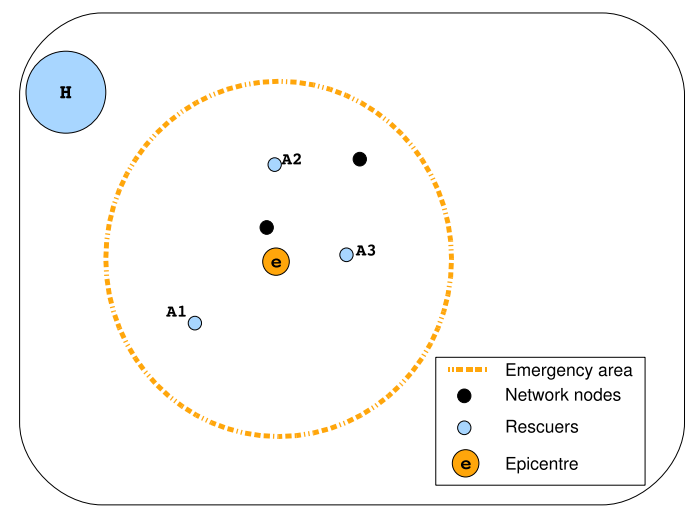

Fig. 1. A mobile ad hoc network in an earthquake area

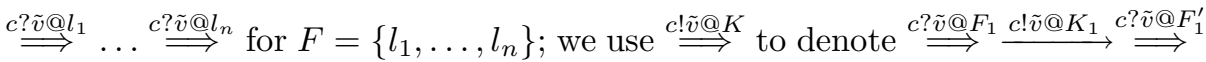
$\ldots \stackrel{c ? \tilde{v} @ F_{n}}{\Longrightarrow} \stackrel{c ! \tilde{v} @ K_{n}}{\Longrightarrow} \stackrel{c ? \tilde{v} @ F_{n}^{\prime}}{\Longrightarrow}$ for $K=\bigcup_{i=1}^{n} K_{i}, F=\bigcup_{i=1}^{n}\left(F_{i} \cup F_{i}^{\prime}\right)$ and $F \cap K=\emptyset$; finally, $\stackrel{\hat{\alpha}}{\Longrightarrow}$ denotes $\Rightarrow$ if $\alpha=\tau$ and $\stackrel{\alpha}{\Longrightarrow}$ otherwise.

Notice that $\stackrel{c ! \tilde{\tilde{\omega}} @ K}{\Longrightarrow}$ means that a distributed observer receiving an instance of message $\tilde{v}$, at each location in $K$, in several computational steps, cannot assume that those messages belong to the same broadcast transmission, but they may be different transmissions of the same message. The presence of the weak input actions $\stackrel{c ? \tilde{v} @ F_{i}}{\Longrightarrow}$ are due to the fact that we want to ignore all the inputs executed by each location which is not included in the set of the intended receivers.

Definition 3 (Labelled bisimilarity). A binary relation $\mathcal{R}$ over networks is a simulation if $M \mathcal{R} N$ implies:

- If $M \stackrel{\alpha}{\longrightarrow} M^{\prime}, \alpha \neq c$ ? $\tilde{v} @ l$, then there exists $N^{\prime}$ such that $N \stackrel{\hat{\alpha}}{\Longrightarrow} N^{\prime}$ with $M^{\prime} \mathcal{R} N^{\prime}$;

- If $M \stackrel{c ? \tilde{v} @ l}{\longrightarrow} M^{\prime}$ then there exists $N^{\prime}$ such that either $N \stackrel{c ? \tilde{v} @ l}{\Longrightarrow} N^{\prime}$ with $M^{\prime} \mathcal{R} N^{\prime}$ or $N \Rightarrow N^{\prime}$ with $M^{\prime} \mathcal{R} N^{\prime}$.

We say that $N$ simulates $M$ if there is some simulation $\mathcal{R}$ such that $M \mathcal{R} N$. A relation $\mathcal{R}$ is a bisimulation if both $\mathcal{R}$ and its converse are simulations. Labelled bisimilaty, written $\approx$, is the largest bisimulation over networks.

Labeled bisimilarity is a valid proof method for reduction barbed congruence.

Theorem 1. Let $M$ and $N$ be two well-formed networks. Then, $M \cong N$ if and only if $M \approx N$.

\section{Properties of Mobile Ad Hoc Networks}

In this section we use CMN\# to define and prove some useful properties of mobile ad hoc networks. 
We consider a running example, depicted in Figure 1, describing the case of an emergency due to an earthquake. The hospital $(\mathrm{H})$ sends three ambulances (A1, A2, A3) to the emergency area. An ad hoc network is installed to manage the communication between the ambulances, placing a router near the epicenter (e) of the earthquake.

We assume that for each process $P$ executed by a network node, it is possible to identify the set of all the intended recipients that may appear in an output action performed by $P$. We denote by $\operatorname{rcv}(P)$ the minimum set of locations ensuring that for each output action $\bar{c}_{L}\langle\tilde{w}\rangle$ performed by $P$ it holds that $L \subseteq$ $\operatorname{rcv}(P)$. Indeed, the tag $L$ associated to an output action occurring in $P$ can be either a variable or a set of locations, then we are not able to statically calculate $\operatorname{rcv}(P)$. However, since an ad hoc network is usually designed to guarantee the communications within a specific area, we can reasonably assume that the underling protocol will always multicast messages to recipients located within the interested area and we can abstractly represent them by a finite set of locations.

Radius of maximum observability. We can define a "radius of maximum observability", that is a radius ensuring the correct reception of a message from all the locations in the recipients set. In particular, we define the "minimum radius of maximum observability", which corresponds to the distance between the sender of the message and the most distant recipient. Clearly, this property is relevant only for stationary nodes, since mobile nodes can always move within the transmission cell of the transmitter to receive the communication.

Consider two different static devices lying at the same location and executing the same code, but with different power capacities. The behaviour of those devices will be different only if they are able to reach distinct sets of intended receivers. The next theorem shows that if a device can reach all its intended receivers, then it behaves as every other device lying at the same location and executing the same code, but with a larger transmission radius.

Theorem 2 (Radius of maximum observability). Let $n[P]_{l, r}^{\mathrm{s}}$ be a stationary node located at $l$ such that $\operatorname{rcv}(P)=L$ and $d(l, k) \leqslant r_{n}$ for all $k \in L$. Then $n[P]_{l, r}^{\mathrm{s}} \approx m[P]_{l, r^{\prime}}^{\mathrm{s}}$ for every node $m$ such that $r_{m} \geq r_{n}$. In this case, we say that $r_{n}$ is a radius of maximum observability for $n[P]_{l, r}^{\mathrm{s}}$.

The minimum radius of maximum observability can be defined as the smallest radius a node can choose in order to reach all its intended recipients.

Definition 4 (Minimum radius of maximum observability). Let $n[P]_{l, r}^{\mathbf{s}}$ be a stationary node such that $\operatorname{rcv}(P)=L$ and $r_{n}$ is a radius of maximum observability for $n[P]_{l, r}^{\mathrm{s}}$. A radius $r^{\prime}$ is said the minimum radius of maximum observability for $n[P]_{l, r}^{\mathrm{s}}$ if $r^{\prime} \leq r_{n}$ and for all $k \in L$ it holds that $d(l, k) \leq r^{\prime}$ and for all $r^{\prime \prime}<r^{\prime}$ there exists $k^{\prime} \in L$ such that $d\left(l, k^{\prime}\right) \geq r^{\prime \prime}$.

In our example, we can assume that the earthquake has damaged a delimited area and the rescuers need to communicate only within this emergency area. We can then determine the minimum transmission radius which ensures the central 
server of the hospital to be able to communicate with the ambulances sent for assistance in the disaster area.

The notion of minimum radius of maximum observability is relevant when dealing with the problem of power saving, since it provides us a with way of reducing the transmission power of a node without loosing connectivity within the whole network.

Simulation of stationary nodes in different locations. The tag $L$ associated to each output action allows us to express a property of simulation for stationary devices in different locations. Indeed, two stationary nodes, placed at different locations (with therefore different neighbors), but communicating with the same set of intended recipients, result to be observational equivalent.

Theorem 3 (Simulation of stationary nodes at different locations). Let $n[P]_{l_{n}, r}^{\mathbf{s}}$ and $m[P]_{l_{m}, r^{\prime}}^{\mathbf{s}}$ be two stationary nodes located at $l_{n}$ and $l_{m}$, respectively. Assume $\operatorname{rcv}(P)=L, K=\left\{k \mid d\left(l_{n}, k\right) \leq r_{n} \wedge k \in L\right\}$ and $K^{\prime}=\left\{k \mid d\left(l_{m}, k\right) \leq\right.$ $\left.r_{m} \wedge k \in L\right\}$. It holds that

1. If $K^{\prime} \subseteq K$, then $n[P]_{l_{n}, r}^{\mathrm{s}}$ simulates $m[P]_{l_{m}, r^{\prime}}^{\mathrm{s}}$;

2. If $K=K^{\prime}$, then $n[P]_{l_{n}, r}^{\mathrm{s}, r} \approx m[P]_{l_{m}, r^{\prime}}^{\mathrm{s}}$.

This property is useful, e.g., to minimize the number of routers within a network area while ensuring the correct communication between a given set of locations. If two different routers result to exhibit the same behaviour, then one of them can be turn off, thus allowing us to save both power and physical resources.

Range repeaters. Range repeaters are devices which regenerate a network signal in order to extend the range of the existing network infrastructure. Here we generalize the definition of repeater given in [4] and introduce a notion of complete range repeater. We consider range repeaters with both one and two channels.

Definition 5. Let $c$ and $d$ be two channels, $l_{r r}$ be a fixed location, $r_{r r}$ be a transmission radius and $L$ be a set of locations. A repeater with two channels

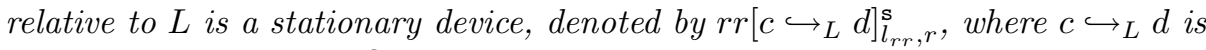
the recursive process defined by:

$$
c \hookrightarrow_{L} d \stackrel{\text { def }}{=} c(x) \cdot \bar{d}_{L}\langle x\rangle \cdot c \hookrightarrow_{L} d .
$$

A range repeater with two channels receives values through the input channel and retransmits them through the output channel. A range repeater with one channel operates likewise, but input and output channels coincide.

Range repeaters are usually exploited to enlarge the transmission cell of a stationary node and, if such a node always communicates with the same set of devices, each time through the same channel, by using a range repeater we can simulate the presence of the sender in the location of the repeater. In our running example, if we consider the distance between the hospital and the earthquake area, we may have that this is too large to guarantee the correct communication with the ambulances running up in the emergency area. It could be necessary to employ a range repeater powerful enough to cover all the area and, at the same 


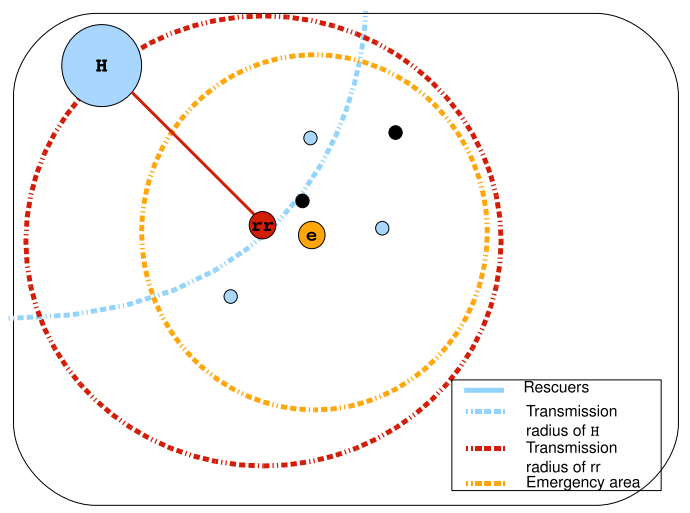

Fig. 2. A range repeater in the earthquake area

time, to be reachable by the central server of the hospital. If the earthquake epicenter is too distant from the hospital we can install a series of consecutive repeaters, which will connect the central server to the disaster area.

Theorem 4 (Range repeaters with one channel). Let $n[P]_{l, r}^{\mathrm{s}}$ be a stationary node such that $f c(P) \subseteq\{c\}$ for some channel $c$ and $\operatorname{rcv}(P)=L$. Let $\operatorname{rr}\left[c \hookrightarrow_{L} c\right]_{l_{r r}, r^{\prime}}^{\mathrm{s}}$ be a range repeater with $d\left(l, l_{r r}\right) \leq r_{n} \leq r_{r r}$. Then:

$$
n[P]_{l, r}^{\mathrm{s}} \mid \operatorname{rr}\left[c \hookrightarrow_{L} c\right]_{l_{r r}, r^{\prime}}^{\mathrm{s}} \text { simulates } n[P]_{l_{r r}, r}^{\mathbf{s}} .
$$

The simulation just described can be realized also with a two-channels range repeater. Using two channels, however, two range repeaters are needed, one for input (in $\left[d \hookrightarrow_{L} c\right]_{l, r^{\prime}}^{\mathrm{s}}$ ) and one for output (out $\left[c \hookrightarrow_{L} d\right]_{l, r^{\prime \prime}}^{\mathrm{s}}$ ) management.

Theorem 5 (Range repeaters with two channels). Let $n[P]_{l, r}^{\mathrm{s}}$ be a stationary node such that $f c(P) \subseteq\{c\}$ for some channel $c$ and $\operatorname{rcv}(P)=$ L. Let in $\left[d \hookrightarrow_{L} c\right]_{k, r^{\prime}}^{\mathrm{s}}$ and out $\left[c \hookrightarrow_{L} d\right]_{k, r^{\prime \prime}}^{\mathrm{s}}$ be two range repeaters with $d(l, k) \leq r_{\mathrm{in}} \leq$ $r_{n} \leq r_{\text {out }}$. Then:

$$
n[P]_{l, r}^{\mathrm{s}}\left|\operatorname{in}\left[d \hookrightarrow_{L} c\right]_{k, r^{\prime \prime}}^{\mathbf{s}}\right| \text { out }\left[c \hookrightarrow_{L} d\right]_{k, r^{\prime}}^{\mathbf{s}} \text { simulates } n[P\{d / c\}]_{k, r}^{\mathbf{s}} .
$$

We introduce the notion of complete range repeater, that is a repeater which has a radius large enough to reach all its intended recipients.

Definition 6 (Complete range repeater). A range repeater $r c\left[c \hookrightarrow{ }_{L} c\right]_{l_{r c}, r}^{\mathbf{s}}$ is said complete with respect to $L$ if $L \subseteq K$ where $K=\left\{k: d\left(l_{r c}, k\right) \leqslant r_{r c}\right\}$.

Consider our running example and suppose that a repeater is installed to allow the central server of the hospital to communicate with the ambulances dispatched in the emergency area (see Figure2). The repeater can be chosen in such a way to guarantee that its transmission radius covers the complete area of the disaster. This is an example of a complete range repeater, whose radius is a radius of maximum observability for the entire earthquake area. 
Theorem 6 (Complete range repeaters). Let $n[P]_{l, r}^{\mathrm{s}}$ be a stationary node such that $f c(P) \subseteq\{c\}$ for some channel $c$ and $\operatorname{rcv}(P)=$ L. Let $r c\left[c \hookrightarrow_{L} c\right]_{l_{r c}, r^{\prime}}^{\mathrm{s}}$ be a complete range repeater with respect to $L$ and $d\left(l, l_{r c}\right) \leq r_{n}$. Then all the recipients in $L$ are reachable by $n$, i.e., $\forall k \in L$, it holds that $d(k, l) \leqslant\left(r_{n}+r_{r c}\right)$.

\section{Conclusion}

Ad hoc networks is a new area of mobile communication networks that has attracted significant attention due to its challenging research problems. Many researchers have proposed formal models, such as a process calculus, in order to reason on their properties and problems (see, e.g., 81356]).

In this paper we extended CMN by associating a tag to each transmission; the tag represents a set of nodes (message receivers) and enables us to prove some important properties concerning the observability of transmissions. For example we have defined the minimum radius of maximum observability, that is the minimum radius ensuring that a packet is reachable by all its receivers; moreover we proved that, under some specific conditions, stationary nodes lying in different locations might have the same behavior. Such properties can be used to reduce the transmission power of a node without loosing connectivity within the whole network.

\section{References}

1. Ad hoc on-demand distance vector routing protocol, http://moment.cs.ucsb.edu/AODV

2. Chakeres, I.D., Belding-Royer, E.M.: Aodv routing protocol implementation design. In: Proc. of 24th International Conference on Distributed Computing Systems Workshops - W7: EC (ICDCSW'04), vol. 7, pp. 698-703. IEEE Press, Los Alamitos (2004)

3. Godskesen, J.C.: A calculus for mobile ad hoc networks. In: Murphy, A.L., Vitek, J. (eds.) COORDINATION 2007. LNCS, vol. 4467, pp. 132-150. Springer, Heidelberg (2007)

4. Merro, M.: An observational theory for mobile ad hoc networks. Information and Computation 207(2), 194-208 (2009)

5. Nanz, S., Hankin, C.: A framework for security analysis of mobile wireless networks. Theoretical Computer Science 367(1), 203-227 (2006)

6. Prasad, K.V.S.: A calculus of broadcasting systems. Science of Computer Programming 25(2-3), 285-327 (1995)

7. Royer, E.M., Perkins, C.E.: Multicast operation of the ad-hoc on-demand distance vector routing protocol. In: Proc. of the 5th annual ACM/IEEE International Conference on Mobile Computing and Networking, pp. 207-2018 (1999)

8. Singh, A., Ramakrishnan, C.R., Smolka, S.A.: A process calculus for mobile ad hoc networks. In: Lea, D., Zavattaro, G. (eds.) COORDINATION 2008. LNCS, vol. 5052, pp. 296-314. Springer, Heidelberg (2008)

9. IEEE 802.11 official website, http://www.ieee802.org/11

10. Zhang, B., Mouftah, H.T.: Energy-aware on-demand routing protocols for wireless ad hoc networks. Wireless Networks 12(4), 481-494 (2006) 